\title{
Serum levels of interleukin 33 and its receptor ST2 in patients treated with subcutaneous allergen immunotherapy in intermittent allergic rhinitis
}

\author{
JOANNA GLÜCK, BARBARA RYMARCZYK, EDYTA JURA-SZOETYS, BARBARA ROGALA \\ Chair and Clinical Department of Internal Diseases, Allergology, and Clinical Immunology, Medical University of Silesia, Katowice, Poland
}

\begin{abstract}
Introduction: Interleukin 33 (IL-33) is a pleiotropic cytokine involved in pathological processes in seasonal allergic rhinitis. IL-33 binds to ST2 receptor, which is highly expressed on mast cells and selectively on Th2 cells. Information is lacking on the role of IL-33/ST2 axis in allergen subcutaneous immunotherapy.

Aim of the study: To determine if allergen immunotherapy changes the IL-33/ST2l axis in seasonal allergic rhinitis patients.

Material and methods: 40 patients with intermittent allergic rhinitis sensitive to grass and/or tree pollen were studied. Among these, 10 patients were longitudinally assessed in the follow-up visit after completing the first course of immunotherapy. Twenty-two healthy subjects were included as controls. Immunotherapy was applied according to a perennial schedule comprising up-building and maintenance phases. Serum levels of ST2/IL-33 R and IL-33 were measured by ELISA (R\&D Systems).

Results: Serum levels of IL-33 significantly rose after the first course of immunotherapy and reached the controls levels. Serum levels of ST2 were comparable before the pollen season and after the first course of immunotherapy.

Conclusions: Increase in serum levels of IL-33 after the first course of immunotherapy may suggest it is too short period to prevent the expected raise in serum IL-33 levels in the pollen season, and longer treatment is required to observe significant changes of this cytokine. ST2 serum levels are independent of immunotherapy and pollen season.
\end{abstract}

Key words: allergic rhinitis, subcutaneous immunotherapy, ST2, IL-33.

(Centr Eur J Immunol 2019; 44 (2): 214-217)

\section{Introduction}

Allergen immunotherapy is a common method to treat allergic diseases such as seasonal allergic rhinitis. Despite its apparent efficacy, the mechanism of its action remains incompletely understood. Recently, an important role of epithelial-derived interleukin (IL) 1 cytokine, such as IL-33, has been described in health and disease [1-3]. IL-33 is expressed by a variety of immune cells participating in allergic inflammation and is mainly known to induce Th2-type cytokine response [4, 5]. Overexpression of IL-33 has been linked to several allergic diseases such as allergic rhinitis and asthma [6, 7]. IL-33 levels were found to be elevated in serum and exhaled breath condensate but were not detectable in nasal lavages in allergic rhinitis [8]. Down-regulation of IL-33 at mRNA and protein levels following sublingual immunotherapy in a murine model of immunosensitisation was reported [9]. However, information is lacking on the role of the IL-33/ST2 axis in allergen subcutaneous immunotherapy. Herein, we aimed to determine the serum level of IL-33 and ST2 before and after the first course of allergen immunotherapy.

\section{Material and methods}

Forty patients (a group treated with allergen immunotherapy - ITA, 13 female, age: $38.5 \pm 10.35$ years) with intermittent allergic rhinitis sensitive to grass and/or tree pollen were studied. Among these, 10 patients were longitudinally assessed in the follow-up visit after completing the first course of immunotherapy, i.e. after the induction and maintenance phases at the beginning of the pollen

Correspondence: Joanna Glück, PhD, Chair and Clinical Department of Internal Diseases, Allergology, and Clinical Immunology,

Medical University of Silesia, 35 Ceglana St., 40-952 Katowice, Poland, e-mail: joagluck@mp.pl

Submitted: 12.01.2018, Accepted: 14.03.2018 
season. In addition, 22 healthy subjects (16 female, age: $37.4 \pm 7.3$ years) without any signs of allergic or inflammatory diseases were included as controls. Diagnosis of intermittent allergic rhinitis was made according to ARIA criteria [10] followed by immunotherapy according to GA2LEN/EAACI criteria [11]. The study was approved by the Ethics Committee of the Medical University of Silesia, and informed consent was obtained from all study participants. Immunotherapy was applied according to a perennial schedule comprising up-building and maintenance phases as per the manufacturers' recommendations. Preparations containing modified allergen were used (Allergovit ${ }^{\circledR}$; Allergopharma or Purethal, Hall Allergy). Blood samples were collected twice from the ITA patients and once from the controls outside the pollen season to separate serum, which was then stored at $-80^{\circ} \mathrm{C}$ until further analysis. Serum levels of ST2 and IL-33 were measured by ELISA (Human ST2/IL-33 R Quantikine ELISA kit, R\&D Systems) and IL-33 (Human IL-33 Quantikine
ELISA kit, R\&D Systems). Results are expressed as median values with interquartile ranges. Nonparametric tests (Mann-Whitney $U$ and Wilcoxon matched pairs tests) were applied using Quick STATISTICA 12.5; $p$-values less than 0.05 were considered significant.

\section{Results}

Serum levels of IL-33 rose significantly after the first course of immunotherapy and reached the controls levels: $1.98 \mathrm{pg} / \mathrm{ml}(1.34-4.05)$ vs. $27 \mathrm{pg} / \mathrm{ml}$ (13-35) (1.34-9.9), $p=0.02$; Wilcoxon matched pairs test and in controls $13.5 \mathrm{pg} / \mathrm{ml}$ (11-32; Mann-Whitney $U$ test) (see Table 1 , Figs. 1 and 2).

Serum levels of ST2 were comparable before the pollen season and after the first course of immunotherapy: $667 \mathrm{pg} / \mathrm{ml}$ (476-829) vs. $555.5 \mathrm{pg} / \mathrm{ml}$ (486-914), $p$ nonsignificant, Wilcoxon matched pairs test, and to controls: $610 \mathrm{pg} / \mathrm{ml}$, Mann-Whitney $U$ test, $p=$ NS) (Table 1).

Table 1. Serum levels of IL-33 and ST2 before and after the first course of immunotherapy (ITA) and in controls (median and total ranges)

\begin{tabular}{|c|c|c|c|c|}
\hline Cytokine & $\begin{array}{c}\text { ITA } \\
n=40\end{array}$ & $\begin{array}{l}\text { ITA-II } \\
n=10\end{array}$ & $\begin{array}{c}\text { CON } \\
n=22\end{array}$ & $p$ \\
\hline $\mathrm{IL}-33(\mathrm{pg} / \mathrm{ml})$ & $1.98(1.34-4.05)$ & $27(13-55)$ & $13.5(11-32)$ & $\begin{array}{l}0.00001 \text { - ITA vs. CON } \\
\text { NS - ITA-II vs. CON } \\
0.02 \text { - ITA vs. ITA-II }\end{array}$ \\
\hline $\mathrm{ST} 2(\mathrm{pg} / \mathrm{ml})$ & $667(476-829)$ & $555.5(486-914)$ & $610(449-819)$ & NS for all comparisons \\
\hline
\end{tabular}

ITA indicates the intermittent allergic rhinitis group before immunotherapy, ITA-II-intermittent allergic rhinitis group after reaching the maximal dose, CON - control group, NS - not significant

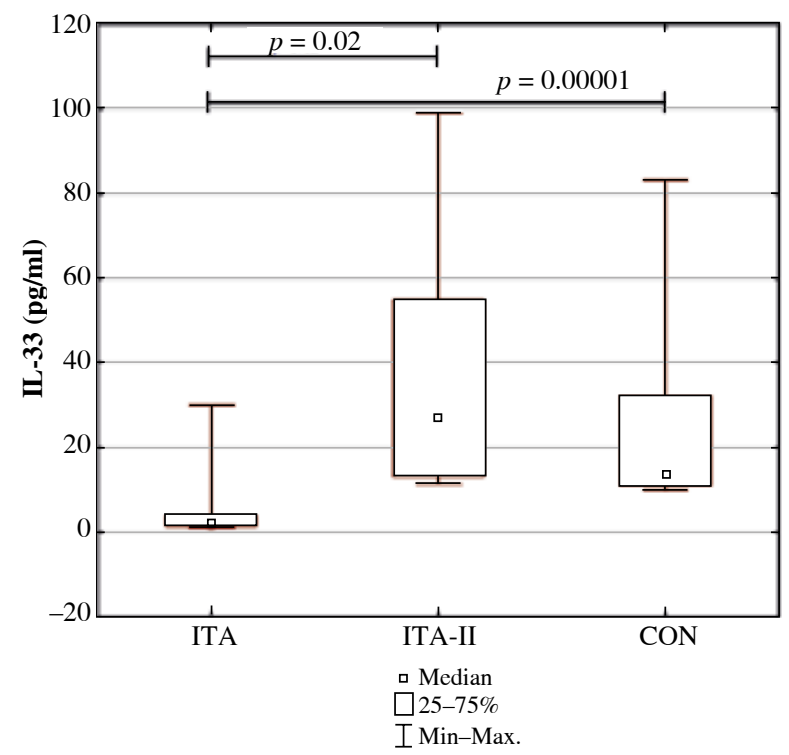

Fig. 1. Serum levels of IL-33 before immunotherapy (ITA) and after the first course of immunotherapy (ITA-II) and in controls (median and total ranges). Only significant differences are shown

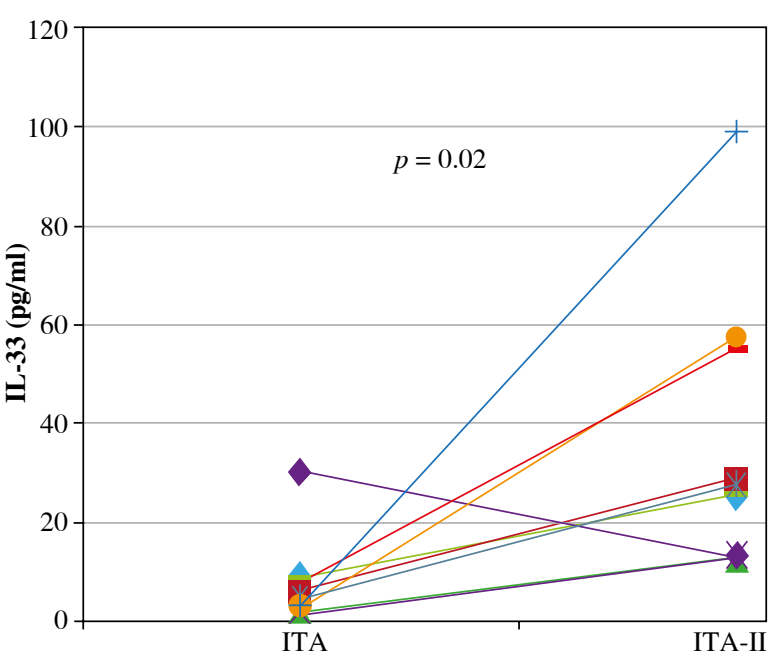

Fig. 2. Serum levels of IL-33 (pg/ml) before immunotherapy (ITA) and after the first course of immunotherapy (ITA-II) 


\section{Discussion}

IL-33 is a pleiotropic cytokine involved in several pathological processes such as allergic diseases. In the present study the serum levels of IL-33 before immunotherapy were lower in patients with intermittent allergic rhinitis before pollen season and rose significantly after the first course of immunotherapy (Figs. 1 and 2). Interestingly, in the present study the serum levels of IL-33 in allergic rhinitis patients before immunotherapy were lower than in the controls, and after the first immunotherapy course they were comparable to the levels in the controls. The results are surprising because we expected IL-33 to be lower after immunotherapy. Thus, we can assume that one course of pre-seasonal immunotherapy is too short a period to prevent raised serum IL-33 levels. However, a similar phenomenon, i.e. the transient increase in allergy markers in the first phase of immunotherapy, has been observed for e.g. sIgE during both subcutaneous and sublingual pollen immunotherapy followed by blunting of seasonal increases in sIgE levels $[12,13]$.

Another explanation of this findings may be the suggestion that immunotherapy did not change the intensification of allergic pathways in the aspect of the IL-33/ST2 axis. Further studies are warranted to find out the pattern of IL-33 serum level changes in the following years of immunotherapy.

We have previously reported significantly higher serum levels of IL-33 in patients with intermittent allergic rhinitis during natural exposure to pollens than in healthy non-atopic controls [6]. The higher level of IL-33 during pollen season could result from pollen exposure acting as an alarmin signal. IL-33 may be released by epithelial cells during their injury or necrosis caused by exogenous triggers such as airborne allergens or by endogenous triggers $[14,15]$. The longer period of immunotherapy might suppress the production of alarmins such as IL-33, but this needs to be elucidated.

Values of serum levels of IL-33 in our study were low and, in some cases, even below the limit of detection suggested by the manufacturer. In this situation the results were obtained by extrapolation. There are some data in literature suggesting that measurement of IL-33 in serum is difficult and sometimes IL-33 may not be detectable. One of the explanations of this phenomena is interference of ST2 with circulating IL-33 and other technical aspects concerning the specificity and sensitivity of currently available assays [16].

In contrast to IL-33, ST2 serum levels did not change after the course of immunotherapy. Such results are consistent with our previous study [6] in which we proved comparable serum levels of ST2 in patients with intermittent allergic rhinitis sensitised to tree/grass pollen and in healthy controls. On the other hand, Baumann et al. found increased levels of ST2 in nasal lavage in seasonally pol- len exposed allergic volunteers [8], suggesting a regulatory role of IL-33 receptor in allergic rhinitis. Thus, nasal epithelium and nasal lavage are probable more suitable sources of ST2 than serum is.

To our knowledge, there are no other data concerning the IL-33/ST2 axis and immunotherapy in human allergic diseases, highlighting the need for further research in this field. Our study has limitations that need to be considered while interpreting the findings. The first one is the small sample size and the high percentage of drop-out of patients who were qualified to immunotherapy, resulting in a smaller group of patients followed up on the second occasion. The second limitation is the short period of observation of the allergic rhinitis patients, which was only during one course of immunotherapy. Consequently, further studies are warranted to elucidate the role of the IL-33/ST2 axis in allergen immunotherapy targeting intermittent and perennial allergic rhinitis.

\section{Acknowledgments}

The authors gratefully acknowledge technical assistance from Mirosława Kasprzak. The study was supported by grant: KNW-1-149/N/4/0 from the Medical University of Silesia.

The authors declare no conflict of interest.

\section{References}

1. Mehray V, Ponte R, Routy JP (2016): The Dynamic Role of the IL-33/ST2 Axis in Chronic Viral-infections: Alarming and Adjuvanting the Immune Response. EBioMedicine 9: 37-44.

2. Lloyd CM (2010): IL-33 family members and asthma - bridging innate and adaptive immune responses. Curr Opin Immunol 22: 800-806.

3. Ohno T, Morita H, Arae K, et al. (2012): Interleukin-33 in allergy. Allergy 67: 1203-1214.

4. Smithgall MD, Comeau MR, Yoon BR, et al. (2008): IL-33 amplifies both Th1- and Th2-type responses through its activity on human basophils, allergen-reactive Th2 cells, iNKT and NK cells. Int Immunol 20: 1019-1030.

5. Pichery M, Mirey E, Mercier P, et al. (2012): Endogenous IL-33 is highly expressed in mouse epithelial barrier tissues, lymphoid organs, brain, embryos, and inflamed tissues: in situ analysis using a novel IL-33-LacZ gene trap reporter strain. J Immunol 188: 3488-3495.

6. Glück J, Rymarczyk B, Rogala B (2012): Serum IL-33 but not ST2 level is elevated in intermittent allergic rhinitis and is a marker of the disease severity Inflamm Res 5: 547-550.

7. Glück J, Rymarczyk B, Kasprzak M, Rogala B (2016): Increased levels of interleukin-33 and thymic stromal lymphopoietin in exhaled breath condensate in chronic bronchial asthma. Int Arch Allergy Immunol 61: 51-56.

8. Baumann R, Rabaszowski M, Stenin I, et al. (2013): Nasal levels of soluble IL-33R ST2 and IL-16 in allergic rhinitis: inverse correlation trends with disease severity. Clin Exp Allergy 43: 1134-1143. 
9. Pishdadian A, Varasteh A, Gholamin M, et al. (2016): Lung-derived innate cytokines: new epigenetic targets of allergen-specific sublingual immunotherapy. Iran J Basic Med Sci 19: 64-71.

10. Bousquet J, Khaltaev N, Cruz A, et al. (2008): Allergic Rhinitis and its Impact on Asthma (ARIA) 2008. Allergy 63: 8-160.

11. Zuberbier T, Bachert C, Bousquet PJ, et al. (2010): GA2 LEN/EAACI pocket guide for allergen-specific immunotherapy for allergic rhinitis and asthma. Allergy 65: 1525-1530.

12. Shamji MH, Ljorring C, Francis JN, et al. (2012): Functional rather than immunoreactive levels of IgG4 correlate closely with clinical response to grass pollen immunotherapy. Allergy 67: 217-226.

13. Dahl R, Kapp A, Colombo G, et al. (2008): Sublingual grass allergen tablet immunotherapy provides sustained clinical benefit with progressive immunologic changes over 2 years. J Allergy Clin Immunol 121: 512-518.e2.

14. Moussion C, Ortega N, Girard JP (2008): The IL-1-like cytokine IL-33 is constitutively expressed in the nucleus of endothelial cells and epithelial cells in vivo: a novel "alarmin"? PLoS One 3: e3331.

15. Lloyd CM (2010): IL-33 family members and asthma - bridging innate and adaptive immune responses. Curr Opin Immunol 22: 800-806.

16. Ketelaar ME, Nawijn MC, Shaw DE, et al. (2016): The challenge of measuring IL-33 in serum using commercial ELISA: lessons from asthma. Clin Exp Allergy 46: 884-887. 